\title{
QUALITY FACTOR ENHANCEMENT OF IN SITU ANNEALING CANTILEVERS
}

\author{
A. Tewary, K.Y. Yasumura $\dagger$, T.D. Stowe $\ddagger$, T.W. Kenny \\ Department of Applied Physics and Department of Mechanical Engineering, \\ Stanford University \\ Stanford, CA 94305* \\ D. Rugar \\ IBM Almaden Research Center \\ San Jose, CA 95120
}

\begin{abstract}
Ultrathin cantilevers with force resolution in the aN $/ \sqrt{H z}$ range have been used in a variety of microscopy experiments to detect extremely small forces. Such cantilevers face a fundamental limit to their ability to detect small forces: thermomechanical noise. For ultrathin cantilever structures, improvement of the $Q$ is vital to the improvement of force resolution. Heat treatments to remove surface contaminants have demonstrated improvements in the room temperature $Q$. In order to utilize this treatment in actual experiments, in situ selfannealing single-crystal silicon cantilevers have been fabricated. With $100 \mathrm{~mW}$ of applied power, $0.25 \mu \mathrm{m}$ thick "self-annealing" cantilevers have demonstrated a three-fold improvement in their room temperature $Q$. By integrating the thermal noise spectrum of the cantilevers while they are being annealed, it is deduced that the cantilever is heated to $1000 \pm 100 \mathrm{~K}$.
\end{abstract}

\section{INTRODUCTION}

Ultrathin single crystal silicon cantilevers have been used to demonstrate attonewton force resolution [1]. The force resolution of these cantilevers is limited by thermomechanical noise. This noise is a result of the cantilever being in thermal equilibrium with its surroundings. The stronger the coupling to the surrounding heat bath, the larger the force noise of the cantilever oscillator. For a cantilever of width $w$, length $l$, and thickness $t$ at a temperature $T$, the minimum detectable force is given by

$$
F_{\text {min }}=\sqrt{\frac{w t^{2}}{l Q}} \sqrt{k_{B} T B}(E \rho)^{1 / 4}
$$

where $Q$ is the quality factor of the oscillator, $k_{B}$ is Boltzman's constant, $B$ is the measurement bandwidth, $E$ is the Young's modulus of the cantilever material, and $\rho$ is the cantilever mass density. Because of this geometrical dependence on force resolution, effort has focused on creating smaller oscillators while maintaining high $Q$. Prior work on arrays of silicon and silicon nitride cantilevers has shown, however, that $Q$ is linearly dependent on $t$ and independent of $w$ and $l$ [2-4]. Such behavior indicates that surface loss mechanisms dominate the $Q$ of micron to submicron-thick cantilever structures with resonance frequencies in the $1-10 \mathrm{kHz}$ have exhibited quality factors of $10^{4}$ [5]. High frequency, $2000 \AA$-thick paddle-shaped oscillators with resonance frequencies in the $1-10 \mathrm{MHz}$ range have exhibited quality factors of $10^{3}$ [6]. This result is consistent with the

\footnotetext{
* Travel support for A.Tewary has been generously provided by the Transducers Research Foundation and by the DARPA MEMS and DARPA BioFlips programs.; †Now at Iolon Inc., San Jose, CA 95131; $\ddagger$ Now at Light Connect, Inc., Newark, CA 94560
}

observed reduction in quality factor with decreasing oscillator dimensions.

Annealing has been shown by Wang to improve the quality factor of surface micromachined polysilicon resonator structures for use in rf MEMS devices by a factor of six [7]. Integrated heating paths in surface micromachined polysilicon comb-drive resonators were used to improve the $Q$ and to frequency trim the resonators. Additionally, Chui [8] has shown that applying heating power as little as $1 \mathrm{~mW}$ can heat a micromachined cantilever designed for use in thermomechanical data storage to a temperature of $800{ }^{\circ} \mathrm{C}$ in vacuum causing the cantilever to glow in the visible wavelength range. The use of in situ annealing techniques have also been used to desorb surface oxides [9] and to repair damage in silicon due to implantation induced damage [10].

In complex experiments where the cantilevers being used require alignment to other experimental features such as magnets, optical fibers, or microstrip resonators this can present numerous problems if the quality factor is allowed to degrade before the cantilever can be aligned. Additionally, some experiments may be conducted over time scales of days or possibly weeks. It would be impractical to open up the measurement chamber, apply additional heat treatments to the cantilever, and then realign the cantilever to the optical fiber interferometer and experimental system. An ideal method to heat the cantilevers and to prevent adsorbates from recollecting on the cantilever surface would be to heat them within the vacuum chamber just prior to using them. This can be accomplished through the use of in situ "self-annealing" cantilevers.

\section{SELF-ANNEALING CANTILEVERS}

The in situ annealing cantilevers used in these experiments were fabricated at the Stanford Nanofabrication Facility. Figure 1 shows an SEM of a self-annealing cantilever made from single-crystal silicon. The cantilever is connected to the substrate silicon die by two legs. In order to measure the cantilever motion, a target paddle is located between the cantilever legs to which an optical fiber interferometer is aligned. Included into the cantilever design is a current path and two heater regions. The current paths on the cantilever lead to large bonding regions on the cantilever substrate die. Wirebonding to this region allows a current to flow through the cantilever's two heater regions. Figure 2 shows a side view of an annealing cantilever. In this figure the overhanging region of the substrate is clearly visible. This overhang is used to thermally and mechanically isolate the heater regions and the cantilever from the rest of the support die. The two openings in the substrate also help to thermally, electrically, and mechanically isolate the cantilever structure. A closer view of the cantilever base and substrate region is also shown in Fig. 2. The cantilever leg is $0.25 \mu \mathrm{m}$ thick, $200 \mu \mathrm{m}$ long, and $2 \mu \mathrm{m}$ wide while the heater region is $\sim 4 \mu \mathrm{m}$ thick at its thickest region. The cantilever resonance frequency is $5.3 \mathrm{kHz}$ and is clamped at the overhanging substrate. 


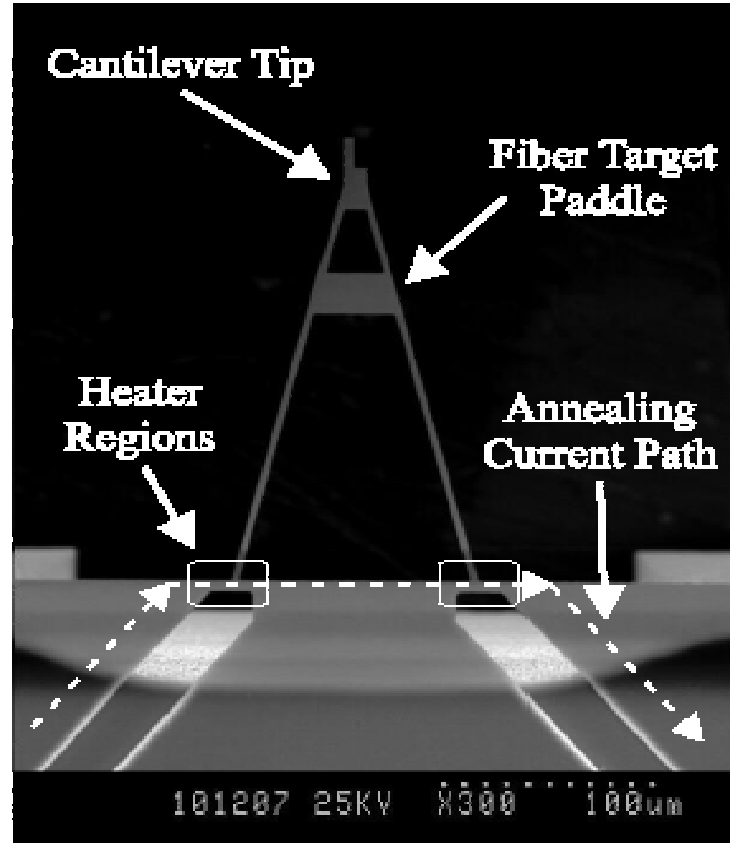

FIG. 1: SEM of a self-annealing cantilever. These cantilevers have a targeting paddle to which the fiber optic interferometer is aligned. Also shown is a current path and two resistive heater regions that warm when an annealing current is applied to the cantilever.
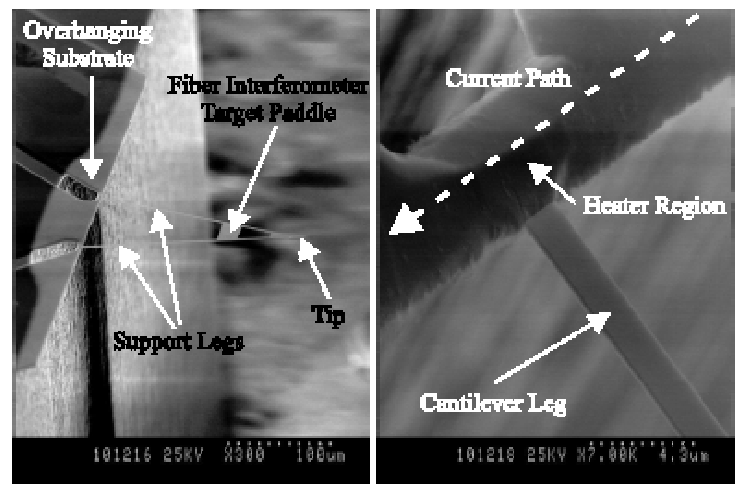

FIG. 2: Side view SEM of an annealing cantilever. The thick overhanging substrate is mechanically isolated from the cantilever, and allows the cantilever to be thermally isolated from the substrate. Also shown is a close-up view of one of the heater regions at the base of a cantilever leg.

The heater regions on the ledge were implanted with boron to a density of $10^{15}-10^{16} \mathrm{~cm}^{-3}$. The cantilever current path was doped to a density of $10^{19} \mathrm{~cm}^{-3}$. A detailed description of the fabrication sequence can be found in Stowe [11].

In order to measure the cantilever motion a $1310 \mathrm{~nm}$ optical fiber interferometer was used [12]. This interferometer had a measured displacement resolution of $2 \times 10^{-3} \AA / \sqrt{\mathrm{Hz}}$. Dissipation measurements were performed using a ring-down technique in which the cantilevers were excited into motion using a self-oscillation circuit. This circuit filtered, amplified, and phase shifted the cantilever's thermal noise signal and then fed this signal to a piezoelectric stack. A current source is used to provide the annealing current to the cantilever under study.

When applying the annealing current to the self-annealing cantilever, care must be taken to avoid thermally shocking the cantilever. The annealing current cannot be turned on and off in a binary fashion to the desired current level. Instantaneously switching on or off the current results in crack formation at the base of the cantilever that causes the resistance to increase and the cantilever leg to burn out. In order to prevent thermally shocking the cantilever, the current source was programmed to provide a gentle rise, current plateau, and then a gentle decrease in the annealing current.

\section{CANTILEVER TEMPERATURE DETERMINATION}

Only a small volume of silicon is warmed when applying the annealing current, thus a direct temperature measurement of the cantilever sample stage will not produce an accurate temperature measurement of the self-annealing cantilever.

In order to obtain a measurement of the cantilever temperature during the high temperature anneal, the thermal noise motion of the cantilever is measured by integration of the thermal noise spectrum of the cantilever [13, 14]. From the equipartition theorem, the amount of thermal energy per mode of a simple harmonic oscillator is $\frac{1}{2} k_{B} T=\frac{1}{2} k\left\langle x^{2}\right\rangle$ where $k_{B}$ is Boltzman's constant, $T$ is the cantilever temperature, $k$ is the cantilever spring constant, and $x$ is the cantilever displacement. Thus, by first measuring the thermal noise displacement at room temperature $(300 \mathrm{~K})$, estimates of the cantilever temperature at higher temperatures can be made because $T_{1} / T_{2}=\left\langle x_{1}^{2}\right\rangle /\left\langle x_{2}^{2}\right\rangle$. The estimates of the cantilever temperature obtained using this method have an uncertainty of $\pm 100 K$. Experiments were performed to anneal the cantilevers at progressively higher temperatures and measure the new resonance frequency and quality factor after the cantilever has been allowed to cool back down to room temperature.

In order to be sure that the temperature that is measured at the base of the cantilever accurately represents the temperature of the entire cantilever, calculations of the estimated thermal conductance of the cantilever can be made. The cantilever shown in Fig. 1 will have power lost due to thermal conduction from the heater regions to the die substrate and from blackbody radiation to its surroundings. It can be shown, however, that the conductance through the overhanging substrate dominates the cantilever equilibrium temperature [15]. Because the experiment is performed in vacuum, thermal convection will not be considered. If an annealing current is applied to the cantilever such that $100 \mathrm{~mW}$ of power is applied at the heater regions heat will flow through the overhanging support to the larger substrate die. From Fig. 1 estimates of the geometry of the conductance path can be obtained. A more accurate calculation would require finite element analysis, but for this purpose it will be shown that estimates are sufficient. The heater region of the cantilever has a thickness of $4 \mu \mathrm{m}$, an estimated length of $10 \mu \mathrm{m}$ to the overhanging substrate region, and a width of $4 \mu \mathrm{m}$. Because there are two leg regions the estimated thermal conductance of the annealing cantilever from the heater region to the substrate die is given by

$$
G=2 \kappa \frac{t w}{l}
$$

where $\kappa$ is the thermal conductivity of silicon at elevated temperatures (at $1000 \mathrm{~K}, \kappa \sim 30 \mathrm{~W} / \mathrm{m} / \mathrm{K}$ ), $t$ is the thickness of the overhanging substrate region, $w$ is the width of the conductance path, and $l$ is the length of the conductance path. With the above values for the annealing cantilever, $G=9.6 \times 10^{-5} \mathrm{~W} / \mathrm{K}$. If $100 \mathrm{~mW}$ of annealing power is applied to the heater regions of the cantilever, the estimated total temperature rise would be $\Delta T=P / G=1040 \mathrm{~K}$. This is in good agreement with the 


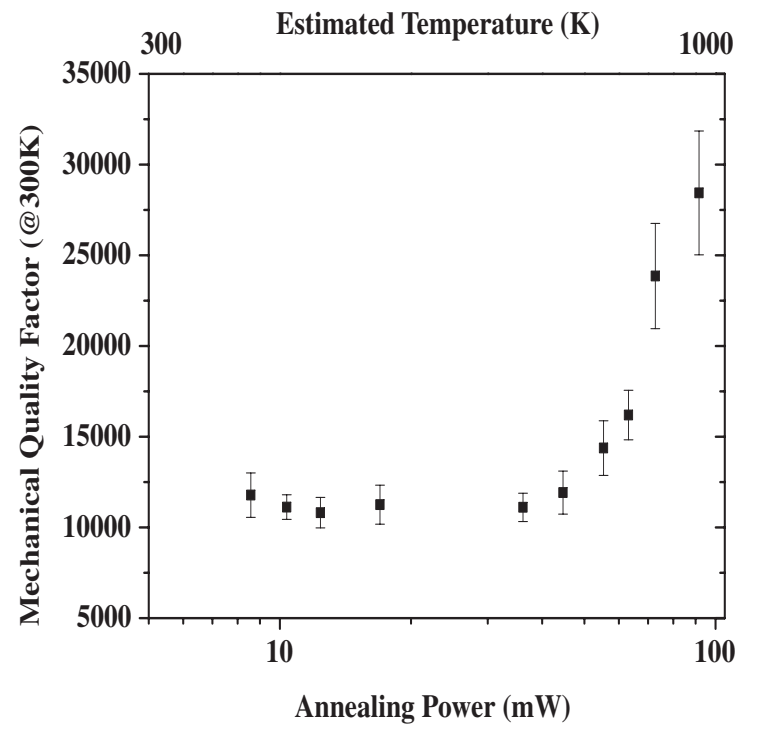

FIG. 3: Room temperature $Q$ after application of an annealing current for a $200 \mu \mathrm{m}$-long cantilever. The cantilever temperature at the highest annealing power was estimated to be $1000 \pm 100 \mathrm{~K}$. This temperature was determined by integrating the cantilever thermal noise.

temperature increase measured from the thermal noise motion for the self-annealing cantilevers.

\section{ANNEALING EXPERIMENTS}

Figure 3 shows a plot of cantilever $Q$ versus annealing power for the $200 \mu \mathrm{m}$-long cantilever that is shown in Fig. 1. The $Q$ measurements were obtained after the cantilever cooled back down to room temperature after each anneal. The values plotted each represent an average of 10 measurements and the error bars are calculated from the standard deviation. A plot of room temperature frequency after that cantilever has been heated at a certain power is shown in Fig. 4. The annealing soak time was 10 minutes for each data point in Figs. 3 and 4 . In this plot, the high temperature was measured by integration of the thermal noise spectrum. The final data point corresponds to an estimated temperature of $1000 \pm 100 \mathrm{~K}$.

In Fig. 3 it is seen that the $Q$ does not start to increase until $\sim 40 \mathrm{~mW}$ of annealing power is applied. This increase in the $Q$ coincides with a large frequency jump $(\sim 20 \mathrm{~Hz})$ observed in the room temperature frequency of the cantilevers. The presence of adsorbed contaminants on the surface of the cantilever causes the resonance frequency to decrease because they add mass to the entire system. Thus, the removal of these contaminants reduces the mass of the system and causes the observed frequency increase when $40 \mathrm{~mW}$ of annealing power is applied. The increase in $Q$ and $f$ are not due solely to the removal of water from the cantilever surfaces. Even though water is removed during the high temperature anneals, it quickly recollects onto the cantilever surface after the annealing current is removed. The easily removed adsorbed contaminants, however, do not quickly return to the cantilever surface resulting in a higher $Q$ value. Possible surface adsorbates include organics introduced in the processing of the cantilevers or during exposure to the laboratory atmosphere. It is not until $100 \mathrm{~mW}$ of annealing power is applied to the cantilever that a three-fold improvement in $Q$ is seen. This applied annealing power corresponds to a temperature of $1000 \pm 100 \mathrm{~K}$, as obtained from the integration

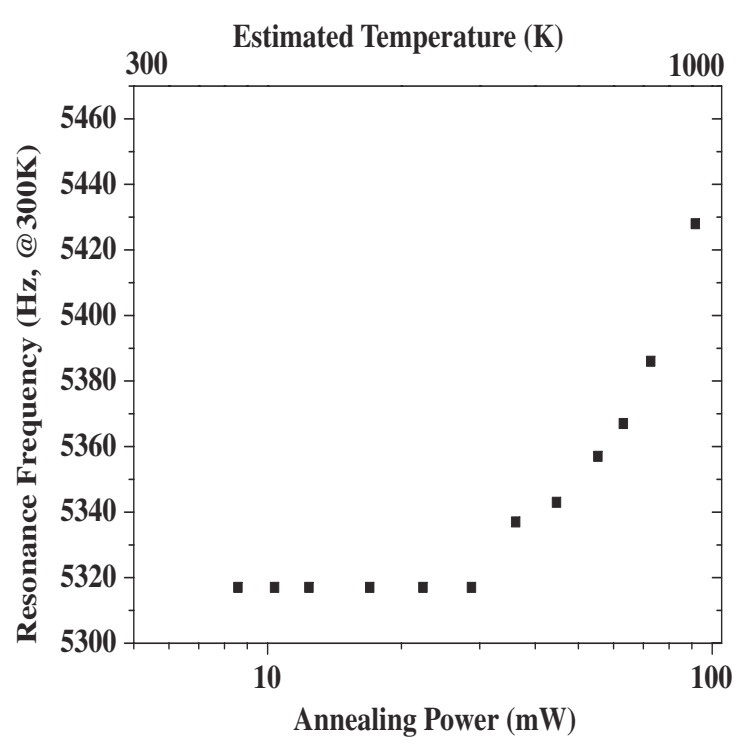

FIG. 4: Room temperature cantilever resonance frequency for a $200 \mu m$-long cantilever, after application of an annealing current that heats the cantilever to the temperature $T$. The cantilever temperature at the highest annealing power was estimated to be $1000 \pm 100 \mathrm{~K}$. This temperature was estimated by integrating the cantilever thermal motion.

of the thermal noise motion. Such high temperatures can begin to desorb oxide from the surface of the silicon cantilevers $[9,16]$.

After the initial annealing experiments, the cantilever was exposed to air then replaced in the vacuum chamber. After the exposure to air, the $Q$ returned to its pre-annealed value. However, the resonance frequency was $14 \mathrm{~Hz}$ less than the preannealed value. Because the spring constant $k$ and the thickness $t$ are related such that $k \propto t^{3}$ and the mass $m$ and the thickness are related such that $m \propto t$, the resonance frequency change is expected to depend linearly with cantilever thickness, since $\omega=$ $\sqrt{k / m} \propto t$. It is therefore expected that as the surface oxide is removed, the thickness of the silicon would decrease thereby resulting in a reduction of the cantilever resonance frequency.

The annealing experiments were conducted on cantilevers of different lengths. Although the experiments were conducted on cantilevers of varying initial quality factors, strong trends are evident. In each case in which the cantilever temperature reached $\sim 1000 \mathrm{~K}$, the $Q$ was enhanced. Furthermore, the resonant frequency of each cantilever was increased by $\sim 3 \%$, which is consistent with mass-loading materials being removed from the surface of the cantilever. The enhanced $Q$ and the resonance frequency shifts were observed in cantilevers that had been stored in air and in cantilevers that had been stored in a dry nitrogen gas environment. Effects of annealing on the $Q$ and resonance frequency for a $350 \mu \mathrm{m}$-long cantilever are shown in Fig. 5 and Fig. 6, and for a $300 \mu \mathrm{m}$-long cantilever are shown in Fig. 7, and Fig. 8. Both of these cantilevers are $0.25 \mu \mathrm{m}$ thick and $2 \mu \mathrm{m}$ wide, and each has a heater region that is $\sim 4 \mu \mathrm{m}$ thick. The $Q$ values plotted represent an average of 10 measurements and the error bars are calculated from the standard deviation. The results indicate that the improvements in quality factor by approximately a factor of three can be seen in cantilevers with varying initial values of $Q$ and resonant frequency.

Arai has studied similar cantilever systems. Arai performed in situ annealing of a silicon piezoresistive cantilever to remove tip contaminants and oxide layers that can reduce image quality when used in an AFM [9]. The piezoresistive AFM cantilever was heated by passing $\mathrm{mA}$ level current through the 


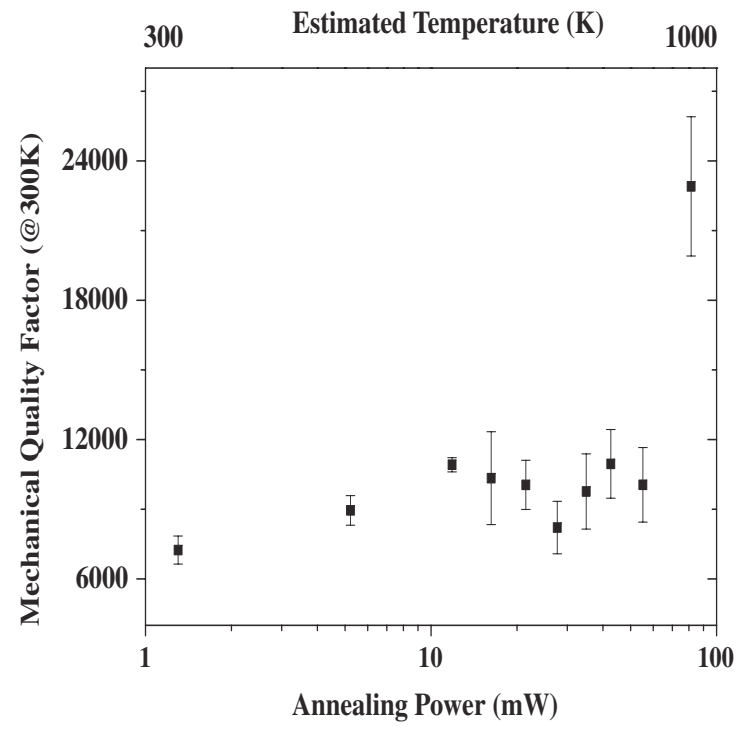

FIG. 5: Room temperature $Q$ vs. Power for $350 \mu m$-long cantilever. The cantilever temperature at the highest annealing power was estimated to be $1000 \pm 100 \mathrm{~K}$. The $Q$ increased from its initial value of $\sim 7000$ to a final value of $\sim 22000$ with the anneal. This cantilever was stored in a dry nitrogen gas environment and had minimal exposure to the laboratory atmosphere. Since additional contaminants were not introduced on the surface, the initial $Q$ is high.

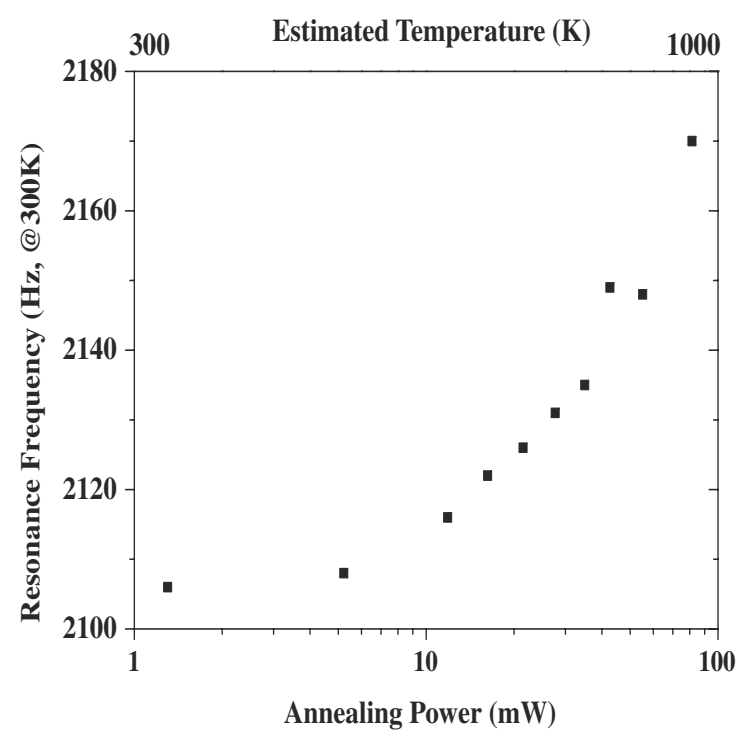

FIG. 6: Room temperature Resonance Frequency vs. Power for $350 \mu$ m-long cantilever. The cantilever temperature at the highest annealing power was estimated to be $1000 \pm 100 \mathrm{~K}$.

resistive regions of the cantilever heating it up to $650{ }^{\circ} \mathrm{C}$. The cantilever temperature was measured using an optical pyrometer with an uncertainty of $\pm 50 \mathrm{~K}$. Arai's experiments were performed at a vacuum chamber pressure of $8 \times 10^{-11}$ torr. Auger electron spectroscopy of the cantilever tip shows that a new cantilever will release $\mathrm{Si}, \mathrm{C}$, and $\mathrm{O}$ in measureable quantities. After annealing at $650{ }^{\circ} \mathrm{C}$ for 1 hour both the $\mathrm{C}$ and $\mathrm{O}$ peaks were removed from the Auger spectra. Although information is not given on how long the cantilever surface remains clean for further oxide and contaminant collection, Arai was

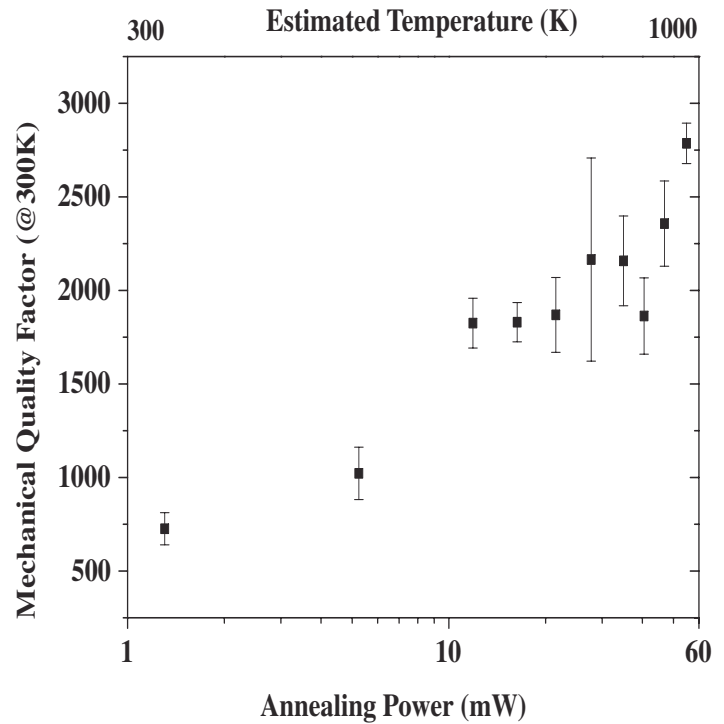

FIG. 7: Room temperature $Q$ vs. Power for $300 \mu m$-long cantilever. The cantilever temperature at the highest annealing power was estimated to be $1000 \pm 100 \mathrm{~K}$. The $Q$ increased from its initial value of $\sim 700$ to a final value of $\sim 2700$. This cantilever was exposed to the laboratory atmosphere for an extended period of time, which may have contributed to the high level of contamination on the surface.

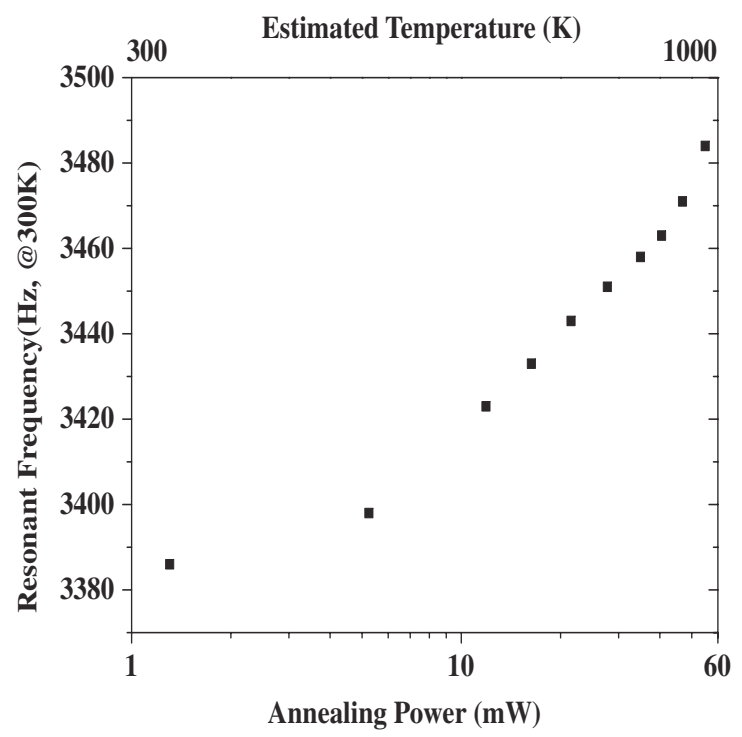

FIG. 8: Room temperature Resonance Frequency vs. Power for $300 \mu \mathrm{m}$-long cantilever. The cantilever temperature at the highest annealing power was estimated to be $1000 \pm 100 \mathrm{~K}$.

able to demonstrated improved AFM spatial resolution after surface treatment. In order to demonstrate control over surface oxide formation, a new oxide surface layer was grown by heating the cantilever to $650{ }^{\circ} \mathrm{C}$ in an $\mathrm{O}_{2}$ environment with a pressure of $5 \times 10^{-5}$ torr. Subsequent Auger spectra analysis showed that an oxide reformed in this manner could be removed by re-annealing the cantilever in the UHV vacuum chamber.

Yang has also studied the effect of in situ heating of singlecrystal silicon cantilevers in vacuum [17]. Yang has demonstrated that significant improvement in $Q$ can be obtained when heating a silicon cantilever in vacuum to $1000{ }^{\circ} \mathrm{C}$ for 30 seconds. 
Yang used x-ray photoelectron spectroscopy to show that annealing at $1000{ }^{\circ} \mathrm{C}$ removed both $\mathrm{C}$ and O-based materials from the cantilever surface.

\section{CONCLUSIONS}

In situ "self-annealing" cantilevers are a novel method to improve the understanding of the surface-related dissipation mechanisms present in ultrathin cantilevers. Our results indicate that the removal of contamination from the surface of the cantilever produces significantly enhanced $Q$ values. However, the removal of easily adsorbed surface contaminants does not appear to significantly influence the quality factor. It is not until temperatures of $\sim 1000 \mathrm{~K}$ are applied that there is a three-fold improvement in the room temperature quality factor for cantilevers with varying initial values of $Q$ and resonant frequency. Our results, and the results of others in the literature, strongly suggest the removal of the oxide from the surface of the cantilever enhances the quality factor.
Possible future experiments include performing these same measurements in a UHV vacuum system in order to be able to control the removal of surface materials in a more precise manner. The addition of residual gas analysis systems would allow determination of the exact contaminant materials liberated from the cantilever surface during the high temperature anneals. Additionally, the use of forming gases to provide additional passivation and surface stability can be used to extend the lifetime of the improved quality factors.

\section{ACKNOWLEDGEMENTS}

This work has been supported by the National Science Foundation CAREER Award (CMR-9971414) and the National SciencE Foundation Instrumentation for Materials Research Program (DMR 9504099). This work also made use of the National Nanofabrication Users Network facilities funded by the National Science Foundation under award number ECS-9731294.
[1] T. Stowe, K. Yasumura, T. Kenny, D. Botkin, K. Wago, and D. Rugar, Appl. Phys. Lett. 71, 288 (1997).

[2] D. Carr, S. Evoy, L. Sekaric, H. Craighead, and J. Parpia, Appl. Phys. Lett. 75, 920 (1999).

[3] K. Yasumura, J. Chiaverini, T. Kenny, and D. Rugar, Transducers 1999 pp. 540-544 (1999).

[4] K. Yasumura, T. Stowe, E. Chow, T. Pfafman, T. Kenny, B. Stipe, and D. Rugar, IEEE J. Microelectromech. Syst. 9, 117 (2000).

[5] K. Yasumura, T. Stowe, E. Chow, T.Pfafman, T. Kenny, and D. Rugar, Solid-State Sensors and Actuator Workshop, Hilton Head, South Carolina, June 8-11, 1999 pp. 65-70 (1998).

[6] S. Evoy, D. Carr, L. Sekaric, A. Olkhovets, J. Parpia, and H. Craighead, J. Appl. Phys. 86, 6072 (1999).

[7] K. Wang, A. Wong, W. Hsu, and C.-C. Nguyen, Transducers 1997 pp. 109-112 (1997).

[8] B. Chui, AFM cantilevers for thermomechanical data storage, Ph.D. Dissertation (1999).

[9] T. Arai and M. Tomitori, Appl. Phys. A 66, S319 (1998).
[10] L. Csepregi, E. Kennedy, J. Mayer, and T. Sigmon, J. Appl. Phys. 49, 3906 (1978).

[11] T. Stowe, Extending the lower limits of force detection using micrommachined silicon cantilevers, Ph.D. Dissertation (2000).

[12] D. Rugar, H. Mamin, and P. Guethner, Appl. Phys. Lett. 55, 2588 (1989)

[13] Y. Martin, C. Williams, and H. Wickramasinghe, J. Appl. Phys. 61, 4723 (1987).

[14] J. Hutter and J. Bechhoefer, Rev. Sci. Instrum. 64, 1868 (1993).

[15] K. Yasumura, Energy Dissipation mechanisms in microcantilever oscillators with application to the detection of small forces, Ph.D. Dissertation (2001).

[16] U. Hashim, S. Shaari, and B. Majlis, ICSE '98 Proc. pp. 213-216 (1998).

[17] J. Yang, T. Ono, and M. Esashi, Appl. Phys. Lett. 77, 3860 (2000). 